\title{
Association between vascular endothelial growth factor and hypertension in children and adolescents type I diabetes mellitus
}

\author{
K Zorena ${ }^{1}$, J Myśliwska ${ }^{1}$, M Myśliwiec $^{2}$, K Rybarczyk-Kapturska ${ }^{1}$, E Malinowska ${ }^{1}$, \\ $\mathrm{P}$ Wiśniewski ${ }^{3}$ and $\mathrm{K}$ Raczyńska ${ }^{4}$ \\ ${ }^{1}$ Department of Immunology, Medical University of Gdańsk, Gdańsk, Poland; ${ }^{2}$ Diabetological Department \\ at Clinic of Paediatrics, Haematology, Oncology and Endocrinology of the Medical University of Gdanisk, \\ Gdańsk, Poland; ${ }^{3}$ Department of Endocrinology and Internal Medicine, Medical University of Gdańsk, Gdańsk, \\ Poland and ${ }^{4}$ Department and Clinic of Ophthalmology, Medical University of Gdańsk, Gdańsk, Poland
}

The aim of the study was to analyse the relationship between the serum level of vascular endothelial growth factor (VEGF) and the incidence of hypertension (HT) in children and adolescents with type I diabetes mellitus (T1DM). One hundred and five patients with T1DM were enrolled in the study. The control group consisted of $\mathbf{3 0}$ healthy controls. All the T1DM patients were subjected to biochemical analyses, ophthalmologic examination and 24-h blood pressure monitoring. Besides, all the patients and healthy controls had serum VEGF levels measured with the use of the ELISA methodology. The essence of our research is that patients with T1DM and HT and with microalbuminuria (MA) and diabetic retinopathy (DR) (MA DR) are characterized by a significantly higher level of VEGF $\left(340.23 \pm 93.22 \mathrm{pg} \mathrm{ml}^{-1}\right)$ in blood serum in comparison with the group of T1DM patients without $\mathrm{HT}$ and MA/DR $\left(183.6 \pm 96.6 \mathrm{pg} \mathrm{m}^{-1}\right)$ and with healthy controls
(145.32 $\left.\pm 75.58 \mathrm{pg} \mathrm{ml}^{-1}\right)$. In addition, the VEGF level was significantly higher in T1DM patients, who presented all three complications, that is $\mathrm{HT}$, retinopathy and MA in comparison with T1DM patients without HT, but with MADDR $(P=0.036)$. On the other hand, no statistically significant differences $(P=0.19)$ were noted in the level of VEGF in serum between T1DM patients without HT and MA/DR and the healthy control group. At a further stage of analysis, using the method of multiple regression, it was shown that systolic pressure, HbA1c and duration of disease are independent factors influencing the concentration of VEGF. Summarizing, the measurement of VEGF serum levels allows for the identification of groups of patients who have the highest risk of HT and, subsequently, progression of vascular complications.

Journal of Human Hypertension (2010) 24, 755-762; doi:10.1038/jhh.2010.7; published online 18 February 2010

Keywords: VEGF; microangiopathy; type I diabetes; children and adolescents

\section{Introduction}

In recent years, a dramatic increase in the incidence of diabetes in developed countries has been observed. ${ }^{1-3}$ Accordingly, also in Poland in the last decade, a significant increase in type I diabetes mellitus (T1DM) incidence has been reported for the paediatric population. ${ }^{4-6}$ Along with increased incidence of diabetes, the risk of development of diabetic microvascular complications, as well as the percentage of patients with arterial hypertension (HT), has also increased. ${ }^{7-9}$ According to various estimations, $1-5 \%$ of children have HT. ${ }^{10,11}$ It has also been shown that elevated blood pressure is $2-3$ times more frequent in diabetic patients as com-

Correspondence: Dr K Zorena, Department of Immunology, Medical University of Gdańsk, Dębinki 1, Gdańsk 80-210, Poland. E-mail: kzorena@gumed.edu.pl

Received 24 July 2009; revised 27 December 2009; accepted 10 January 2010; published online 18 February 2010 pared with the general population. ${ }^{12}$ It is suggested that, in the course of T1DM, the presence of elevated values of arterial blood pressure is associated with the development of diabetic nephropathy and retinopathy. ${ }^{13-15}$ Apart from HbA1c, which is a well-known risk factor for micro- and macrovascular complications, recent data suggest that growth factors, including vascular endothelial growth factor (VEGF), may have an important function in the modification of tissue damage and its acceleration. ${ }^{16-19}$ It was shown that VEGF induces vascular endothelial cell proliferation and migration and increases the permeability of renal glomerular and retinal capillaries. ${ }^{18-20}$

There is solid experimental and clinical evidence of the function of VEGF in retinal angiogenesis. Retinal hypoxia leads to a pronounced increase in VEGF levels in several cell types, including pericytes, Muller cells, astrocytes, retinal endothelial cells and retinal pigment epithelial cells. ${ }^{13,21-25}$ From literature, it is known that the serum VEGF 
level corresponds to its level in the eye structures. Hermandez et al. ${ }^{21}$ have shown that elevated VEGF level in the aqueous humour correlated with increased serum VEGF and was statistically significantly higher in relation to the healthy control group. A pilot study performed by Lip et $a .^{22}$ showed that patients with hypertensive retinopathy had increased serum levels of VEGF and its soluble receptor Flt-1. In our earlier studies, we have documented the significance of VEGF in nonproliferative retinopathy development in children and adolescents with T1DM. ${ }^{23-24}$ Others have also shown the upregulation of VEGF mRNA expression in pericytes as well as podocytes and mezangial cells in the early stages of disease, indicating its function in diabetic angiopathy. ${ }^{20,25}$ The VEGF is the strongest inducer of both physiological and pathological angiogenesis. ${ }^{17,26}$ Five different types, such as VEGF-A, VEGF-B, VEGF-C, VEGF-D, VEGF-E and eight isoforms of this cytokine are known (VEGF121, VEGF145, VEGF148, VEGF162, VEGF165, VEGF183, VEGF189, VEGF206). The VEGF isoforms differ in the length of the amino-acid chain, mitogenic activity and the VEGF receptors and heparin-binding affinities. ${ }^{19,26,27}$ The VEGF exerts its biological effects by binding to two surface tyrosine kinase receptors. The VEGF receptor-1 (VEGFR-1) and VEGF receptor-2 (VEGFR-2) are predominantly expressed on vascular endothelial cells. The VEGF receptor-2 seems to be the main mediator of angiogenesis and vasopermeability. ${ }^{27}$

Adult HT is easily defined by sustained blood pressure $>140 / 90 \mathrm{~mm} \mathrm{Hg}$, whereas no single cutoff point defines HT in a paediatric patient, making identification of childhood HT more difficult. ${ }^{28,29}$ According to the Second National Heart, Lung and Blood Institute Task Force, HT is defined as an average systolic and/or average diastolic blood pressure $\geqslant 95$ th percentile for age, sex and height measured on at least three separate occasions. ${ }^{30,31}$ Till now, the only available tool for predicting HT was 24-h ambulatory blood pressure monitoring. Hence, our study is aimed to search for early risk factors of microangiopathy with HT, which would become useful for the diagnosis of complication in young patients with diabetes type I. Given that HT is one of the most important factors influencing the development of late vascular complications, we have decided to analyse the levels of VEGF in children and adolescents diagnosed with T1DM and HT in relation to diabetes control and other clinical parameters of the disease.

\section{Material and methods}

The study was carried out on 105 children and adolescents with T1DM from the Department of Paediatrics, Haematology, Oncology and Endocrinology of the Medical University of Gdańsk and on 30 healthy children and adolescents as a control group. T1DM was defined in accordance with the criteria of the American Diabetes Association. ${ }^{32}$

\section{Laboratory examinations}

HbA1c was measured with an immunoturbidometric method using Unimate 3 set (Hoffmann-La Roche AG, Basel, Switzerland) with a normal range of values $3.0-6.0 \%$. Fasting glucose was measured by enzymatic test (Roche Diagnostics GmbH, Mannheim, Germany). The level of CRP was measured with immunochemical system (Beckman Instr. Inc., Galway, Ireland). The level of C-peptide was below $0.5 \mathrm{ng} \mathrm{ml}^{-1}$ in all children with T1DM. The urinary albumin excretion was expressed as the average of the three 24-h collections obtained during 6 months before enrolment into the study. Cases were classified as microalbuminuria (MA) when in at least two out of three urine samples albumin excretion was between 30$299 \mathrm{mg}$ per $24 \mathrm{~h}$. The urinary albumin excretion is measured by immunoturbidometric assay using Tinaquant (Boehringer Mannheim GmbH, Mannheim, Germany). The serum level of creatinine was measured with the use of CREA assay system (Boehringer Mannheim $\mathrm{GmbH}$ ). Ophthalmologic investigation was performed in all children with T1DM. This included visual acuity tests, intraocular pressure and anterior segment estimation by slit lamp (Topcon SL-82, Tokyo, Japan). The fundus examination was performed after the installation of $1 \%$ Tropicamid to obtain sufficient mydriasis. The examination was performed using the $+90 \mathrm{D}$ lens (Ocular Instruments, Inc, Bellevue, WA, USA). A digital camera (Topcon Imaginet 2000, Tokyo, Japan) was used to perform the fluorescein angiography. Analysis of the eye fundus pictures was based on The International Diabetic Retinopathy Division. ${ }^{33}$

Each patient was subjected to threefold 24-h ambulatory blood pressure monitoring by the Holter method. Various sizes of the cuff were used according to the age and weight of the patient. The apparatus was programmed to measure two time spans: daily (600-2200 hours) and nocturnal (2200600 hours). All the ambulatory blood pressure monitoring results, which had $<80 \%$ of technically correct measurements were excluded from the study. In accordance with the report, The fourth report on the diagnosis, evaluation and treatment of high blood pressure in children and adolescents, arterial HT was diagnosed when the blood pressure value reached at least 95th percentile for the corresponding age, gender and height on at least three separate occasions. ${ }^{31}$

The study was conducted on four groups: patients with long-standing T1DM and diagnosed to have HT and MA/diabetic retinopathy (DR) $(n=16)$; diabetic patients without signs of HT and MA/DR $(n=74)$; diabetic patients without HT, but with MA/DR $(n=15)$ and a healthy control group $(n=30)$. The clinical and biochemical parameters of the analysed groups are summarized in Table 1. 
Table 1 Clinical characteristics of T1DM patients and healthy subjects

\begin{tabular}{|c|c|c|c|c|c|}
\hline & \multirow{2}{*}{$\begin{array}{l}\text { T1DM patients with } \\
\text { HT with MA/DR }\end{array}$} & \multicolumn{2}{|c|}{ T1DM patients without $H T$} & \multirow{2}{*}{$\begin{array}{l}\text { Healthy } \\
\text { subjects }\end{array}$} & \multirow[t]{2}{*}{$\mathrm{P}$} \\
\hline & & $\begin{array}{l}\text { Without } \\
\text { MA/DR }\end{array}$ & $\begin{array}{l}\text { With } \\
M A / D R\end{array}$ & & \\
\hline$n$ & 16 & 74 & 15 & 30 & \\
\hline Age (years) & $17 \pm 4$ & $15.8 \pm 2.9$ & $16.6 \pm 3.6$ & $16 \pm 4.0$ & $\begin{array}{l}P=0.034^{*} \\
P=\mathrm{NS}^{* *} \\
P=0.36^{* * *}\end{array}$ \\
\hline Diabetes onset (years) & $7.3 \pm 3.3$ & $7.6 \pm 3.6$ & $7.2 \pm 3.9$ & - & $\begin{array}{l}P=\mathrm{NS}^{*} \\
P=\mathrm{NS}^{* *}\end{array}$ \\
\hline Duration of DM (years) & $9.3 \pm 3.9$ & $7.3 \pm 2.2$ & $9.4 \pm 3.2$ & - & $\begin{array}{l}P=0.0001^{*} \\
P=\mathrm{NS}^{* *}\end{array}$ \\
\hline HbA1C (\%) & $9.5 \pm 2.3$ & $7.7 \pm 1.0$ & $8.7 \pm 1.4$ & $4.2 \pm 0.3$ & $\begin{array}{l}P=0.0001^{*} \\
P=0.02^{* *} \\
P=0.00001^{* * *}\end{array}$ \\
\hline $\mathrm{CRP}\left(\mathrm{mg} \mathrm{l}^{-1}\right)$ & $1.6 \pm 1.0$ & $0.9 \pm 0.7$ & $1.5 \pm 1.0$ & $0.7 \pm 0.5$ & $\begin{array}{l}P=0.0001^{*} \\
P=\mathrm{NS}^{* *} \\
P=0.16^{* * *}\end{array}$ \\
\hline Albumin excretion rate (mg per $24 \mathrm{~h}$ ) & $20.6(3.1-115.2)$ & $9.0(0.9-28.8)$ & $31.5(7.5-141.2)$ & $2.8 \pm 1.2$ & $\begin{array}{l}P=0.0001^{*} \\
P=\mathrm{NS}^{* *} \\
P=0.000001^{* * *}\end{array}$ \\
\hline Creatinine in serum $\left(\mu \mathrm{moll}^{-1}\right)$ & $0.8 \pm 0.2$ & $0.78 \pm 0.1$ & $0.77 \pm 0.1$ & $0.5 \pm 0.1$ & $\begin{array}{l}P=\mathrm{NS}^{*} \\
P=\mathrm{NS}^{* *} \\
P=0.0001^{* * *}\end{array}$ \\
\hline $\mathrm{SBP}(\mathrm{mm} \mathrm{Hg})$ & $129.0 \pm 7.0$ & $108.0 \pm 8.0$ & $110.0 \pm 9.0$ & $110.0 \pm 8.0$ & $\begin{array}{l}P=0.0001^{*} \\
P=0.008^{* *} \\
P=0.001^{* * *}\end{array}$ \\
\hline $\mathrm{DBP}(\mathrm{mm} \mathrm{Hg})$ & $83.0 \pm 9.0$ & $68.0 \pm 6.0$ & $70.0 \pm 8.0$ & $65.0 \pm 7.0$ & $\begin{array}{l}P=0.0006^{*} \\
P=0.0003^{* *} \\
P=0.0001^{* * *}\end{array}$ \\
\hline VEGF $\left(\mathrm{pg} \mathrm{ml}^{-1}\right)$ & $340.23 \pm 93.22$ & $183.6 \pm 96.6$ & $247.3 \pm 138.6$ & $145.3 \pm 75.6$ & $\begin{array}{l}P=0.035^{*} \\
P=0.036^{* *} \\
P=0.00000^{* * *}\end{array}$ \\
\hline Complications (n) & 16 & - & 15 & - & $0.05^{*}$ \\
\hline Hypertension & 16 & - & - & - & - \\
\hline Microalbuminuria & 15 & - & 10 & - & $0.03^{*}$ \\
\hline $\begin{array}{l}\text { Non-proliferative retinopathy } \\
\text { and microalbuminuria }\end{array}$ & 16 & - & 5 & - & $0.00001^{*}$ \\
\hline
\end{tabular}

Abbreviations: DBP, diastolic blood pressure; DM, diabetes mellitus; DR, diabetic retinopathy; HT, hypertension; MA, microalbuminuria; SBP, systolic blood pressure; T1DM, type I diabetes mellitus; VEGF, vascular endothelial growth factor.

Data are presented as means \pm s.d., albumin excretion rate as (min and max).

${ }^{*}$ Group with T1DM with HT and with MA/DR vs group without HT and MA/DR.

${ }^{* *}$ Group with T1DM with HT and MA/DR vs group without HT and with MA/DR.

*** Group with T1DM with HT and MA/DR vs healthy subjects.

All patients with T1DM required insulin treatment $\left(0.87 \pm 0.24 \mathrm{U} \mathrm{kg}^{-1}\right.$ of the body weight). Thirty healthy children and adolescents volunteered as the control group, after medical examination. Written informed consent was obtained from all children and adolescents participating in the study, or from a parent or guardian.

This study was approved by The Ethics Committee of The Medical University of Gdańsk (NKEBN/ 204/2009) and the investigation was carried out in accordance with the principles of the Declaration of Helsinki as revised in 1996.

\section{Sample collection}

Plasma samples were collected from 135 children and adolescents. Blood samples were immediately placed on ice, clarified by centrifugation at $3.000 \mathrm{~g}$ for $5 \mathrm{~min}$ at $4{ }^{\circ} \mathrm{C}$, and kept frozen at $-80^{\circ} \mathrm{C}$ until assayed.
Serum level of VEGF

Serum level of VEGF ${ }_{165}$ was measured by the ELISA method (Quantikine High Sensitivity Human by R\&D System, Minneapolis, Minn., USA) in accordance with the manufacturer's protocol. Minimum detectable concentrations were determined by the manufacturer as $5.0 \mathrm{pg} \mathrm{ml}^{-1}$. Intra-assay precision was 53.7 and inter-assay $91 \%$ were determined on 20 replicates from the quality control data of the laboratory.

\section{Statistical analysis}

All statistical analysis was undertaken with the Statistica 8.0 software (StatSoft, Inc., Cracow, Poland). The Shapiro-Wilk test was used to evaluate normality of variables. Non-normal variables were compared with non-parametric methods, that is the Mann-Whitney $U$ or the Kruskal-Wallis ANOVA. 
Correlations were examined using the Spearman's rank order method. Normal variables were analysed using parametric methods, that is the Student's $t$-test, ANOVA, Pearson's correlation.

To investigate factors influencing serum VEGF concentration, a general linear model was fitted. The model included age, sex, HbA1c, duration of disease, albumin excretion rate, systolic and diastolic blood pressure as the independent variables. In all analyses, a two-tailed significance level of $<0.05$ was regarded as statistically significant.

\section{Results}

Clinical and biochemical parameters of patients with T1DM

The group of T1DM patients with HT and MA/DR was older and with a longer duration of the disease, a higher level of HbA1c, higher C-reactive protein, a higher daily excretion of albumin and higher pressure, both systolic and diastolic in comparison with the group of patients without HT and MA/DR. On the other hand, when comparing the same group of T1DM patients with HT and without MA/DR with the group without HT, but with MA/DR, no statistically significant differences were shown in age, duration of the disease, CRP level or daily excretion of albumin. Statistically significant differences were shown only in the level of HbA1c and in systolic and diastolic pressure.

In addition, when comparing the group of T1DM patients with HT onto the healthy group, significantly higher levels of HbA1c, CRP, albumin extraction rate and elevated systolic and diastolic blood pressure were observed than in healthy subjects (Table 1).

\section{Levels of VEGF in T1DM patients}

The highest level of VEGF was in group T1DM patients with HT and MA/DR as compared with T1DM patients without HT and MA/DR $(P=0.035)$ as well as T1DM patients without HT, but with MA/ DR $(P=0.036)$. In addition, a statistically significantly higher level of VEGF was observed in T1DM patients with HT and MA/DR as compared with the healthy subjects $(P=0.0000)$ (Table 1$)$. Moreover, a statistically significantly lower $(P=0.001)$ serum level of VEGF was observed in T1DM patients without HT and MA/DR in comparison with T1DM patients without HT, but with MA/DR. On the other hand, when comparing the group of T1DM patients without HT and MA/DR to the control group, we did not note any statistically significant differences $(P=0.19)$ in the level of VEGF (Figure 1).

Univariate correlation between systolic and diastolic blood pressure and VEGF level in T1DM patients In T1DM patients, we observed a statistically significant correlation between systolic blood pres- sure and serum VEGF level (Spearman $r=0.4$; $P=0.00004$ ) (Figure 2) as well as between systolic blood pressure and HbA1c level (Spearman $r=0.5$; $P=0.000002$ ) (Figure 3 ) and a correlation of low significance between diastolic blood pressure and serum VEGF level (Spearman $r=0.2 ; P=0.041$ )

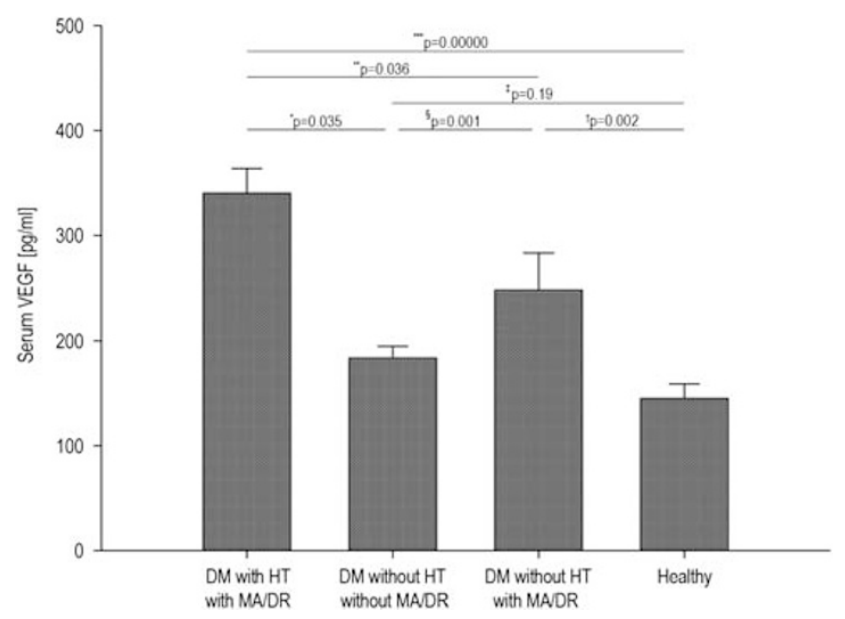

Figure 1 Levels of VEGF in T1DM patients. DM with HT with MA/DR-diabetes mellitus with HT and MA and/or DR. DM without HT and MA/DR_-diabetes mellitus without HT and MA and/or DR. DM without HT and with MA/DR-diabetes mellitus without HT and with MA and/or DR. The figure represents significantly higher level of VEGF patients with T1DM and HT and with MA/DR vs T1DM patients without HT and MA/DR $\left({ }^{*} P=0.035\right)$ and significantly higher level compared with diabetes mellitus (DM) group without HT and with MA/DR $\left({ }^{*} P=\right.$ $0.036)$ as well as with the healthy subjects $\left({ }^{* *} P=0.00000\right)$. Moreover, it represents significantly $(\$ P=0.001)$ lower level of VEGF in DM group without HT and MA/DR vs DM without HT and with MA/DR, as well as lack of significance $\left({ }^{\ddagger} P=0.19\right)$ in comparison with the healthy group.

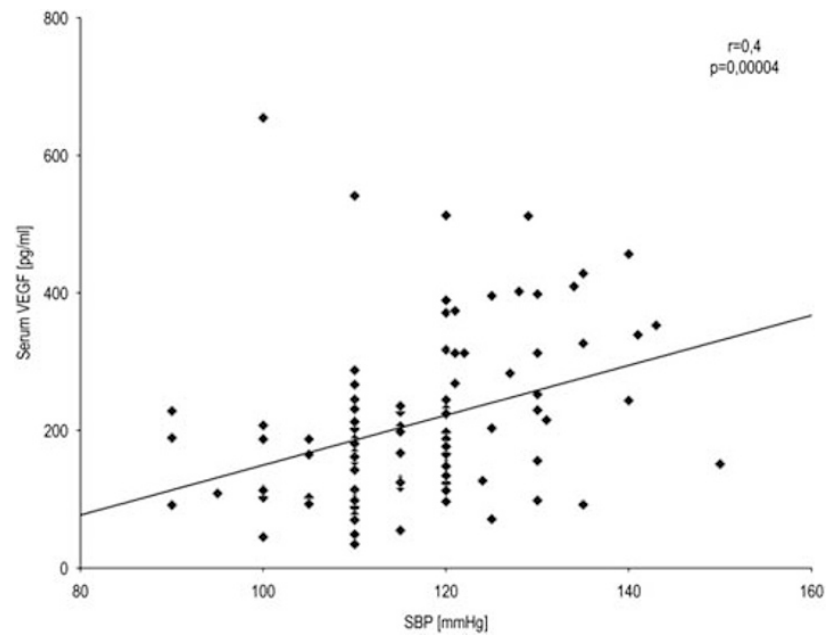

Figure 2 Correlation between systolic blood pressure and VEGF level in T1DM patients. A statistically significant correlation between systolic blood pressure and serum VEGF was observed. Spearman's rank correlation $(r=0.4, P=0.0004)$ for all T1DM patients. 


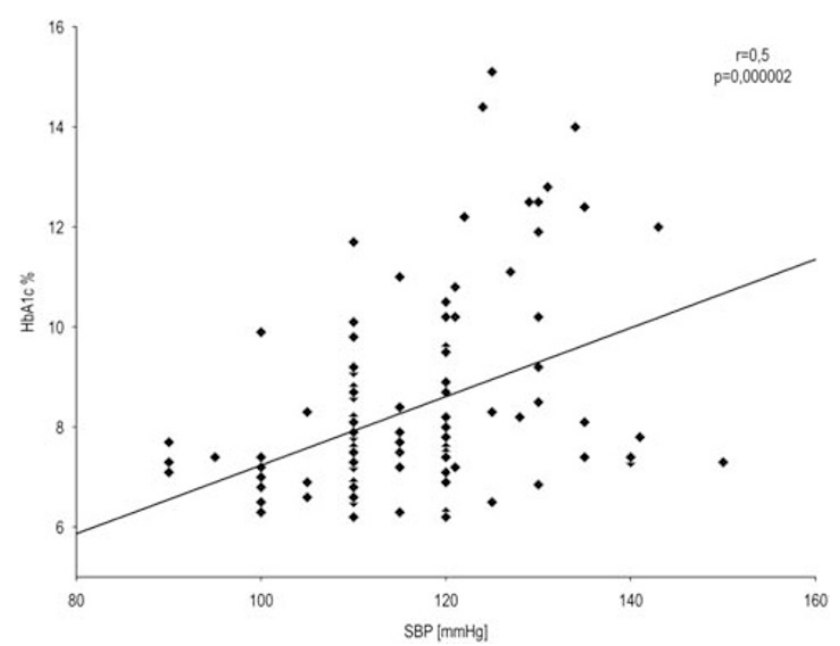

Figure 3 Correlation between systolic blood pressure and HbA1c level in T1DM patients. A statistically significant correlation between systolic blood pressure and HbA1c level $(r=0.5$, $P=0.000002)$ was observed. Spearman's rank correlation $(r=0.5, P=0.000002)$ for all T1DM patients.

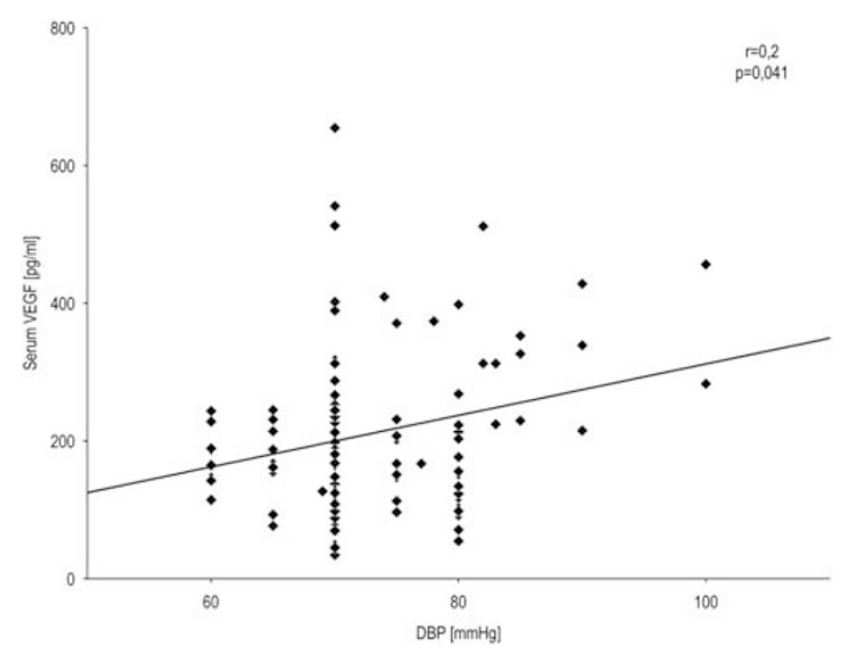

Figure 4 Correlation between diastolic blood pressure and VEGF level in T1DM patients. The correlation between diastolic blood pressure and serum VEGF level was calculated. Spearman's rank correlation $(r=0.2, P=0.041)$ for all T1DM patients.

(Figure 4). There were no significant correlations between other baseline variables.

\section{Multivariate analysis}

To better assess the possible relationship between VEGF and the other variables, a multiple regression model (general linear model based) was created. The model included VEGF level as the dependent variable and the independent variables were age, sex, duration of disease, HbA1c, albumin excretion rate, systolic and diastolic blood pressure. The results of the analysis are displayed in Table 2 . After adjustment for possible confounders, only
Table 2 Results of multiple regression analysis with VEGF as the dependent variable (adjusted $R^{2}=0.30 ; P<0.001$ )

\begin{tabular}{lrrrr}
\hline DV:VEGF & $\beta$ & $-95 \% C I$ & $+95 \% C I$ & P-value \\
\hline HbA1c & 0.22 & 0.03 & 0.42 & 0.026 \\
MA & 0.08 & -0.13 & 0.29 & 0.439 \\
Age & 0.08 & -0.17 & 0.32 & 0.531 \\
SBP & 0.28 & 0.04 & 0.53 & 0.022 \\
DBP & 0.06 & -0.15 & 0.28 & 0.551 \\
Duration of DM & 0.20 & 0.00 & 0.40 & 0.050 \\
Sex & -0.11 & -0.29 & 0.07 & 0.231 \\
\hline
\end{tabular}

Abbreviations: DBP, diastolic blood pressure; DM, diabetes mellitus; Duration of DM, duration of disease; DV, dependent variable; $\beta$, standard coefficient of regression; 95\%CI, 95\% confidence interval for $\beta$; MA, microalbuminuria; SBP, systolic blood pressure; VEGF, vascular endothelial growth factor.

systolic blood pressure $(\beta=0.28 ; P=0.022)$, HbA1c $(\beta=0.22 ; \quad P=0.026)$ and duration of diabetes $(\beta=0.20 ; \quad P=0.050)$ proved to be independent factors influencing serum VEGF concentration. This model was statistically significant and explained $30 \%$ of total VEGF variation in examined patients.

\section{Discussion}

The core of our research is that our patients with T1DM with HT and MA/DR really do reveal a higher level of VEFG in serum compared with T1DM patients who do not have HT, as well as in comparison with the healthy control group. In addition, the VEGF level was significantly higher in T1DM patients who presented all three complications that is HT, retinopathy and MA in comparison with T1DM patients without HT, but with retinopathy and MA. However, no statistically significant difference was found on the VEGF level in serum between the T1DM group without HT and MA/DR and the healthy control group. These data indicate that the VEGF level is not significantly higher in these patients, compared with the healthy control group, as long as patients with type I diabetes have not developed any complications. Moreover, patients with MA, retinopathy and HT have the highest level of VEGF secretion, whereas the lowest secretion appears in patients who have no complications. Our results indicate that VEGF may be a potential mediator in the early phase of microvessel lesion in the history of HT in children and adolescents with type I diabetes. Diabetic pathological changes in the microcirculation of the retina, renal glomerule and nerve vessels have a similar pathogenic basis. ${ }^{13,15,18}$

However, despite vast research conducted, there is no unanimous agreement as to the frequency and reasons for HT in children with type I diabetes who have developed microangiopathy. It is known that, on the one hand, HT accelerates and reinforces 
micro- and macrovascular complications in diabetes; on the other hand, in patients with type I diabetes who suffer from diabetic nephropathy, arterial HT is considered to be the secondary result of kidney lesion. ${ }^{34,35}$ In recent years, the necessity for the 24-h measurement of blood pressure in patients with T1DM has been raised to monitor and discover early changes in the blood pressure profile, manifested by the drop of blood pressure during the night, which may be the first symptom of microangiopathy. ${ }^{34,36,37}$

Our earlier research, as well as publications from other authors, ${ }^{16,21-24}$ indicates that VEGF can be an important factor influencing complications related to microangiopathy in patients with type I diabetes; however, never have we met any report on the relation between VEGF and HT in the course of diabetic microangiopathy of children and adolescents suffering from T1DM. Our current research indicates a considerably different interchangeable higher level of HbA1c and systolic pressure in patients with MA, retinopathy and HT, next to a considerably higher level of VEGF, as compared with patients who also suffer from MA and retinopathy, but have no HT. A similar conclusion may be drawn from the research of Torchinsky et al., ${ }^{38}$ who have shown that, in the group of patients with poor metabolic control of diabetes, an increase in arterial blood pressure is observed at an early stage of the disease with consequent incidence of MA. It is know that VEGF synthesis occurs in podocytes in the glomerular and tubular cells as well as collecting channels and VEGF receptors are present in glomerular endothelial cells. ${ }^{20,39}$ Recent in vitro studies have shown a significant increase in VEGF levels, synthesized in the podocytes in high glucose patients. ${ }^{20}$ An increased VEGF in the glomerular endothelial cells may contribute to the development of MA by increasing glomerular permeability through various mechanisms in diabetic patients. ${ }^{20,40}$

Recently, researchers have indicated in their works that MA is not only the symptom of developing diabetic nephropathy, but, also lesions in the organ of vision. It is suggested that in patients with T1DM, the elevated values of arterial blood pressure are associated with the development of DR independently of incipient nephropathy. ${ }^{7,9}$ Such a scenario has also been postulated by large-scale studies, such as, for example, the prospective study by Gallego et al. carried out on a group of adolescents with juvenile onset of type I DM who were affected by the development of early retinopathy. These authors have postulated that both systolic and diastolic blood pressures are predictors of retinopathy and increase the probability of early retinopathy independently of incipient nephropathy in young patients with T1DM. ${ }^{9}$

Our research additionally reveals the correlation between systolic pressure and the VEGF level. The results of multiple regression analysis indicate that systolic blood pressure is an equally important independent variable, influencing VEGF, as HbA1c and the duration of the disease are.

It has not been fully discovered how HT leads to the increased production of VEGF in T1DM patients. It is believed that this is a multi-directional process. On the one hand, it is known that persistent hyperglycaemia, leading to the elevation of HbA1c level, may lead to the production and accumulation of advanced glycation end-products. The formation of advanced glycation end-products promotes production of pro-inflammatory cytokines, which may further initiate the increase of VEGF level, thus indirectly leading to the development of HT. It was proved that VEGF combines with the VEGFR-2 receptor on the surface of endothelium cells, which leads to the phosphorylation of transcription factors by MAPK kinase (miogen-activated protein kinase). ${ }^{17,25}$ As a result of this process, there occurs an increased expression of adhesive molecules, along with an increase in cytokines and chemokines, which foster the proliferation of endothelium cells and the production of an extracellular matrix and at the same time impair its degradation. ${ }^{19-20}$ Consequently, the increasing fibrosis of vessels appears, the vascular lumen decreases, so the blood flow through the vessels is hindered. The increased resistance in the blood system further results in the increased production of VEGF and fosters changes in the structure and proportion of collagen and elastine, thus leading to higher rigidity of vessels and aggravated HT.

Summing up, the results of our research indicate that VEGF is an important factor in the pathogenesis of microangiopathy in cases of this type of HT. Paediatricians are interested in receiving therapeutic tools for young patients with diabetes, aiming at limited complications on the microvascular level. Rivard et al..$^{41}$ attempted to use the methods of gene therapy using adeno-VEGF in non-obese mice (NOD). They reported that inhibition of VEGF expression may contribute to the inhibition of late diabetic complications or to their complete remission. Besides, Fernandez et $a l^{42}$ showed on an animal model that therapy aimed at inhibition of VEGF and PDGF has a significant function in the inhibition of portal HT, causing its reduction even by $40 \%$. In addition, the results of first clinical studies with the participation of T1DM patients are encouraging and maybe in the near future medications inhibiting VEGF will become the principle of the therapy of microangiopathy. ${ }^{43-45}$ Nevertheless, before this becomes true, it is important to monitor VEGF levels in a larger group of patients, which would allow for the identification of a subgroup of patients with the highest risk of the incidence of HT and subsequently late diabetic complications. VEGF may become a marker useful in identification of the patients who in the future might develop HT before the occurrence of the first clinical signs of HT. 
What is known about this topic

- In children, hypertension is usually asymptomatic; therefore, early detection of HT.

- VEGF has an important part in neoangiogenesis with microangiopathy patients.

What this study adds

- VEGF blood serum test in children and adolescents type I diabetes mellitus allows for the isolation of a group of patients with a higher risk of vascular complications.

- VEGF measurement in children and adolescents type I diabetes mellitus may become an extra marker in prognostication of hypertension and microangiopathy.

\section{Conflict of interest}

The authors declare no conflict of interest.

\section{Acknowledgements}

We thank Tadeusz Z Wolański for helpful reading of our paper. The study was financed by the Medical University of Gdańsk (grants ST-28 to Jolanta Myśliwska and ST-56 to Krystyna Raczyńska).

\section{References}

1 Catanzariti L, Faulks K, Moon L, Waters AM, Flack J, Craig ME. Australia's national trends in the incidence of Type 1 diabetes in 0-14-year-olds, 2000-2006. Diabet Med 2009; 26: 596-601.

2 Johnson JA, Vermeulen SU, Toth EL, Hemmelgarn BR, Ralph-Campbell K, Hugel G et al. Increasing incidence and prevalence of diabetes among the Status Aboriginal population in urban and rural Alberta, 19952006. Can J Public Health 2009; 100: 231-236.

3 Patterson CC, Dahlquist GG, Gyürüs E, Green A, Soltész G, EURODIAB Study Group. Incidence trends for childhood type 1 diabetes in Europe during 1989-2003 and predicted new cases 2005-20: a multicentre prospective registration study. Lancet 2009; 373 : 2027-2033.

4 Kretowski A, Kowalska I, Peczyńska J, Urban M, Greek A, Kinalska I. The large increase in incidence of Type I diabetes mellitus in Poland. Diabetologia 2001; 44(Suppl 3): B48-B50.

5 Myśliwiec M, Balcerska A, Zorena K, Jedrzejczyk A, Malinowska E, Myśliwska J. Increasing incidence of diabetes mellitus type 1 in children-the role of environmental factors. Polish J Environ Stud 2007; 16: 109-112.

6 Jarosz-Chobot P, Polańska J, Polański A. Does socialeconomical transformation influence the incidence of type 1 diabetes mellitus? A Polish example. Pediatr Diabetes 2008; 9: 202-207.

7 da Costa Rodrigues T, Pecis M, Azevedo MJ, Esteves JF, Gross JL. Ambulatory blood pressure monitoring and progression of retinopathy in normotensive, normoalbuminuric type 1 diabetic patients: a 6-year follow-up study. Diabetes Res Clin Pract 2006; 74: 135-140.

8 Dursun H, Bayazit AK, Cengiz N, Seydaoglu G, Buyukcelik M, Soran $M$ et al. Ambulatory blood pressure monitoring and renal functions in children with a solitary kidney. Pediatr Nephrol 2007; 22: 559-564.

9 Gallego PH, Craig ME, Hing S, Donaghue KC. Role of blood pressure in development of early retinopathy in adolescents with type 1 diabetes: prospective cohort study. BMJ 2008; 337: a918.

10 Suláková T, Janda J. Ambulatory blood pressure in children with diabetes 1. Pediatr Nephrol 2008; 23: 2285-2286.

11 Kollias A, Antonodimitrakis P, Grammatikos E, Chatziantonakis N, Grammatikos EE, Stergiou GS. Trends in high blood pressure prevalence in Greek adolescents. J Hum Hypertens 2009; 23: 385-390.

12 Palta M, LeCaire T. Managing type 1 diabetes: trends and outcomes over 20 years in the Wisconsin Diabetes Registry cohort. WMJ 2009; 108: 231-235.

13 Silva KC, Pinto CC, Biswas SK, de Faria JB, de Faria JM. Hypertension increases retinal inflammation in experimental diabetes: a possible mechanism for aggravation of diabetic retinopathy by hypertension. Curr Eye Res 2007; 32: 533-541.

14 Kumar P, Krishna P, Reddy SC, Gurappa M, Aravind SR, Munichoodappa C. Incidence of type 1 diabetes mellitus and associated complications among children and young adults: results from Karnataka Diabetes Registry 1995-2008. J Indian Med Assoc 2008; 106: 708-711.

15 Giunti S, Barit D, Cooper ME. Mechanisms of diabetic nephropathy: role of hypertension. Hypertension 2006; 48: 519-526.

16 Ayerden Ebinç F, Haksun E, Ulver DB, Koç E, Erten Y, Reis Altok $\mathrm{K}$ et al. The relationship between vascular endothelial growth factor (VEGF) and microalbuminuria in patients with essential hypertension. Intern Med 2008; 47: 1511-1516.

17 Wirostko B, Wong TY, Simó R. Vascular endothelial growth factor and diabetic complications. Prog Retin Eye Res 2008; 27: 608-621.

18 Aiello LP, Wong JS. Role of vascular endothelial growth factor in diabetic vascular complications. Kidney Int Suppl 2000; 58: 113-119.

19 Kvanta A. Ocular angiogenesis: the role of growth factors. Acta Ophthalmol Scand 2006; 84: 282-288.

20 Iglesias-de la Cruz MC, Ziyadeh FN, Isono M, Kouahou M, Han DC, Kalluri R et al. Effects of high glucose and TGF-beta1 on the expression of collagen IV and vascular endothelial growth factor in mouse podocytes. Kidney Int 2002; 62: 901-913.

21 Hermandez C, Burgos R, Canton A, Garcia-Arumi J, Segura RM, Simo R. Vitreous levels of vascular cell adhesion molecule and vascular endothelial growth factor in patients with proliferative diabetic retinopathy: a case-control study. Diabetes Care 2001; 24: 516-521.

22 Lip PL, Belgore F, Blann AD, Hope-Ross MW, Gibson JM, Lip GY. Plasma VEGF and soluble VEGF receptor FLT-1 in proliferative retinopathy: relationship to endothelial dysfunction and laser treatment. Invest Ophthalmol Vis Sci 2000; 41: 2115-2119.

23 Myśliwiec M, Balcerska A, Zorena K, Myśliwska J, Lipowski P, Raczyńska K. The role of vascular endothelial growth factor, tumor necrosis factor alpha and interleukin-6 in pathogenesis of diabetic retinopathy. Diabetes Res Clin Pract 2008; 79: 141-146.

24 Zorena K, Myśliwska J, Myśliwiec M, Balcerska A, Lipowski P, Raczyńska-Woźniak D et al. Modulatory factors responsible for neoangiogenesis in young patients with long-standing diabetes mellitus type 1 . 
Recent Patents Endo. Metab. Immu Drug Disc 2009; 3: 144-149.

25 Suzuma I, Hata Y, Clermont A, Pokras F, Rook SL, Suzuma $\mathrm{K}$ et al. Cyclic stretch and hypertension induce retinal expression of vascular endothelial growth factor and vascular endothelial growth factor receptor-2: potential mechanisms for exacerbation of diabetic retinopathy by hypertension. Diabetes 2001; 50: $444-454$.

26 Fam NP, Verma S, Kutryk M, Stewart DJ. Clinical guide to angiogenesis. Circulation 2003; 108: 2613-2618.

27 Ferrara N, Gerber HP, LeCouter J. The biology of VEGF and its receptors. Nat Med 2003; 9: 669-676.

28 World Health Organization (WHO)/International Society of Hypertension (ISH) statement on management of hypertension. World Health Organization, International Society of Hypertension Writing Group. J Hypertens 2003; 21: 1983-1992.

29 Treatment of Hypertension in Adults With Diabetes. American Diabetes Association. Diabetes Cares 2002; 25: S71-S73.

30 National High Blood Pressure Education Program Working Group on Hypertension Control in Children and Adolescents. Update on the 1987 Task Force Report on High Blood Pressure in Children and Adolescents: a working group report from the National High Blood Pressure Education Program. Pediatrics 1996; 98: 649-658.

31 National High Blood Pressure Education Program Working Group on High Blood Pressure in Children and Adolescents. The fourth report on the diagnosis, evaluation and treatment of high blood pressure in children and adolescent. Pediatrics 2004; 114(Suppl. 24th Report): 555-576.

32 American Diabetes Association. Fallow-up report on the diagnosis of diabetes mellitus. Diabetes Care 2003; 26: $3160-3167$.

33 ETDRS report number 11. Early Treatment Diabetic Retinopathy Study Research Group. Classification of diabetic retinopathy from fluorescein angiograms. Ophthalmology 1991; 98(Suppl. 5): 807-822.

34 Ingelfinger JR. Blood-pressure control and delay in progression of kidney disease in children. $N$ Engl J Med 2009; 361: 1701-1703.

35 Torffvit O, Eriksson JW, Henricsson M, Sundkvist G, Arnqvist HJ, Blohmé $\mathrm{G}$ et al. Early changes in glomerular size selectivity in young adults with type 1 diabetes and retinopathy. Results from the Diabetes Incidence Study in Sweden. J Diabetes Complications 2007; 21: 246-251.
36 Chiarelli F, Trotta D, Verrotti A, Mohn A. Treatment of hypertension and microalbuminuria in children and adolscents with type 1 diabetes mellitus. Pediatr Diabetes 2002; 3: 113-124.

37 Hansen KW, Poulsen PL, Ebbehøj E, Mogensen CE. What is hypertension in diabetes? Ambulatory blood pressure in 137 normotensive and normoalbuminuric Type 1 diabetic patients. Diabet Med 2001; 18: 370-377.

38 Torchinsky MY, Gomez R, Rao J, Vargas A, Mercante DE, Chalew SA. Poor glycemic control is associated with increased diastolic blood presure and heart rate in children with Type 1 diabetes. J Diabetes Complications 2004; 18: 220-223.

39 Bortoloso E, Del Prete D, Dalla Vestra M, Gambaro G, Saller A, Antonucci F et al. Quantitave and qualitative changes in vascular endothelial growth factor gene expression in glomeruli of patients with type 2 diabetes. Eur J Endocrinol 2004; 150: 799-807.

40 Cha DR, Kim NH, Yoon JW, Jo SK, Cho WY, Kim HK et al. Role of vascular endothelial growth factor in diabetic nephropathy. Kidney Int Suppl 2000; 77: S104-S112.

41 Rivard A, Silver M, Chen D, Kearney M, Magner M, Annex B et al. Rescue of diabetes-related impairment of angiogenesis by intramuscular gene therapy with Adeno-VEGF. Am J Pathol 1999; 154: 355-363.

42 Fernandez M, Mejias M, Garcia-Pras E, Mendez R, Garcia-Pagan JC, Bosch J. Reversal of portal hypertension and hyperdynamic splanchnic circulation by combined vascular endothelial growth factor and platelet-derived growth factor blockade in rats. Нераtology 2007; 46: 1208-1217.

43 Oshima Y, SAkaguchi H, Gomi F, Tano Y. Regression of iris neovascularization after intravitreal injection of bevacizumab in patients with proliferative diabetic retinopathy. Am J Ophthalmol 2006; 142: 155-158.

44 Pandya NM, Dhalla NS, Santani DD. Angiogenesis-a new target for future therapy. Vascul Pharmacol 2006; 44: 265-274.

45 Simó R, Hernández C. Intravitreous anti-VEGF for diabetic retinopathy: hopes and fears for a new therapeutic strategy. Diabetologia 2008; 51: 1574-1580.

(a) This work is licensed under the Creative Commons Attribution-NonCommercialNo Derivative Works 3.0 Unported License. To view a copy of this license, visit http://creativecommons. org/licenses/by-nc-nd/3.0/ 